\title{
Diagnóstico nutricional en el paciente oncológico: Importancia y Herramientas para realizarla.
}

\section{Nutritional diagnosis in cancer patients: Importance and Tools to perform it.}

*Correspondencia: dra.rodriguezv@yahoo.com Teléfono [593] 022990800

Conflicto de intereses: Los autores declaran no tener conflictos de intereses.

Fondos: Ver la página 178

Recibido: 15 Mayo 2017 Aceptado: 21 Julio 2018 Publicado: 30 Diciembre 2018

Membrete bibliográfico: Rodríguez D, Frías-Toral E, Santana S. Diagnóstico nutricional en el paciente oncológico: Importancia y Herramientas para realizarla. Rev. Oncol. Ecu 2018;28(3):168-180.

DOI: https://doi.org/10.33821/271

Copyright Rodríguez, et al. Este artículo es distribuido bajo los términos de Creative Commons Attribution License, el cual permite el uso y redistribución citando la fuente y al autor original.

\section{Dolores Rodríguez Veintimilla1*iD), Evelyn Frías-Toral1, Sergio Santana Porbén2.}

1. Instituto Oncológico Nacional "Dr. Juan Tanca Marengo", Solca-GuayaquilEcuador.

2. Hospital Pediátrico Docente Juan Manuel Marquez de La Habana-Cuba.

\section{Resumen}

Debido a la alta incidencia de diferentes patologías oncológicas a nivel mundial y mortalidad relacionada a ellas resulta fundamental conocer formas de prevenir sus complicaciones que muchas veces son decisivas en la sobrevida del paciente. Esto lo evidencia la relación de un $30 \%$ de las muertes por cáncer asociado a cinco factores de riesgo comportamentales y alimentarios. Ya que éstos pueden ser prevenidos, es urgente ahondar en conocimientos al respecto.

Actualmente varios estudios confirman la vital importancia del estado nutricional de los pacientes con cáncer y su impacto positivo en la evolución de la enfermedad. Un diagnóstico e intervención nutricional tempranos aseguran múltiples beneficios, reducción de complicaciones, recuperaciones más rápidas, reducción de estancia hospitalaria, mejor tolerancia a los esquemas terapéuticos, entre otros.

Existen varias herramientas para realizar un correcto cribado nutricional. En este artículo se describirán el Método del Nutritional Risk Screening 2002 y Valoración Global Subjetiva generada por el Paciente.

Palabras Claves: NEOPLASIAS, MORTALIDAD, ESTADO NUTRICIONAL, CRIBADO.

DOI: $10.33821 / 271$

\section{Abstract}

Due to the high incidence of different types of cancer worldwide and mortality related to them, it is essential to know ways to prevent their complications that have a direct impact on the survival of the patient. $30 \%$ of cancer deaths are associated with five behavioral and food risk factors. By deepening knowledge in this regard, we can avoid them. 
Several studies affirm the vital importance of the nutritional status of cancer patients and their positive impact on the evolution of the disease. The early diagnosis and nutritional intervention assure multiple benefits, reduction of complications, faster recoveries, reduction of length of stay at hospitals, better tolerance to cancer treatments, among others.

There are several tools to perform correct nutritional screening. In this article, we will describe the Nutritional Risk Screening 2002 and the Patient-Generated Subjective Global Assessment

Keywords: NEOPLASMS, MORTALITY, NUTRITIONAL STATUS, STRAINING.

\section{Introducción}

El tratamiento Oncológico óptimo tiene las características de integralidad, constituirse con un trabajo de Equipo y ser oportuno. Dentro de este escenario, el reconocimiento del estado nutricional de los pacientes diagnosticados de cáncer es de suma importancia para seleccionar a los pacientes que puedan tolerar mejor un tratamiento adyuvante, así se contribuirá a sostener la integralidad del sistema inmunológico que se ve afectado por la enfermedad y por los tratamientos utilizados [1].

Seguir un protocolo de trabajo es indispensable, conservando el orden de realizar Cribado Nutricional Inicial. Si éste es positivo para Riesgo de Desnutrición o Desnutrición hay que implementar el consejo dietético y la terapéutica específica a cada paciente de acuerdo a varios aspectos que afectarán directamente en el paciente para lograr una intervención exitosa. De los cuales algunos están en relación con ubicación y estadío del cáncer, edad y actividad del paciente, preferencias alimentarias, trastornos de los sentidos del gusto y olfato, utilización o no de suplementos, trastornos del sueño que muchas veces impiden que el paciente se alimente, hábitos intestinales, afectación psicológica, si está o no en tratamiento para el dolor, así como también considerar si el paciente está con una enfermedad muy avanzada que impide el aporte apropiado de nutrientes [2].

El derecho a alimentarse se encuentra a menudo con obstáculos tan fuertes como la carencia de recursos económicos del paciente y su familia que le impiden adquirir aquella prescripción dietética recomendada por el experto en Nutrición. Así, la prescripción de suplementos pasa a ser un acto dramático cuando se carece de los recursos para adquirirlos, ante lo cual es indispensable que el acto de diagnosticar y tratar nutricionalmente a un paciente oncológico debe ser una política de estado [3].

Adquirir conocimientos académicos en torno al tema del Cáncer y su tratamiento, nos conduce al Trabajo de Equipo en el cual el Oncólogo, el Nutricionista, la Enfermera y el Químico Farmacéutico dialoguen en torno a cada caso con la finalidad de no incurrir en errores terapéuticos y como equipo colaboren en esa relación Personal de Salud-Paciente. 


\section{Estadísticas de Cáncer}

El cáncer es una de las primeras causas de muerte en todo el mundo. En el año 2015 se atribuyeron a esta enfermedad 8,8 millones de defunciones. Los cinco tipos de cáncer que causan un mayor número de fallecimientos son los siguientes: Pulmonar, Hepático, Colorrectal, Gástrico y mamario [4]. Aproximadamente un 30\% de las muertes por cáncer se deben a cinco factores de riesgo comportamentales y alimentarios: Índice de masa corporal elevado, ingesta insuficiente de frutas y verduras $[5,6]$, sedentarismo, tabaquismo y consumo de Alcohol. Se debe destacar que estos cinco factores pueden ser prevenidos, de ahí la importancia que se capacite y eduque a la comunidad.

Cabe también anotar que las infecciones que pueden provocar cáncer, como las causadas por los virus de las hepatitis B y C y el del papiloma humano, son responsables del $20 \%$ de las muertes por cáncer en los países de ingresos bajos y medianos y del $7 \%$ en los países de ingresos altos [5].

En un suplemento de la revista Cáncer Epidemiology [7] se recopilan datos provenientes de 17 artículos científicos, basados en la información aportada por 48 registros de cáncer en 18 países, de América Latina coordinados por la Agencia Internacional para la Investigación del Cáncer (IARC). El estudio incorpora datos de aproximadamente 546 mil nuevos casos ( $47 \%$ hombres, $53 \%$ mujeres) y más de 2 millones de muertes por cáncer ( $52 \%$ hombres, $48 \%$ mujeres) ocurridos entre 2003 y 2007 . El cáncer de próstata, mama, cuello de útero, pulmón, colorrectal y estómago suman el $63 \%$ de los casos y $49 \%$ de las muertes. Países como Argentina y Brasil registraron una alta prevalencia de cáncer de próstata y de mama, mientras que Bolivia y Perú, tuvieron una alta prevalencia de cáncer de cuello de útero y de estómago. Así Latinoamérica presenta una doble prevalencia de cáncer, con tasas elevadas tanto para el cáncer asociado a infecciones que tradicionalmente afecta a las regiones más pobres del mundo como para el cáncer asociado a estilos de vida, que tradicionalmente se asocia con regiones más prósperas [5].

El Proyecto GLOBOCAN estimó que en 2012 hubo 1.000 .000 de casos de cáncer (excluyendo cáncer de piel no melanoma) y medio millón de muertes por cáncer en América Central y del Sur, y predijo para el 2030 estos números serán 1.7 y 1 millón respectivamente [4].

\section{Terapia Nutricional}

Se define como tal al Tratamiento basado en la nutrición. Incluye comprobar el estado nutricional de una persona y dar los alimentos o nutrientes apropiados para tratar afecciones tales como las causadas por la diabetes, la cardiopatía y el cáncer. Puede abarcar cambios sencillos en el régimen alimentario de una persona, o alimentación intravenosa o con sonda. La terapia nutricional médica puede ayudar a que los pacientes se recuperen más rápido y pasen menos tiempo en el hospital [8]. 


\section{Evaluación y Tratamiento Nutricionales en los Hospitales}

En relación a la evaluación y tratamiento Nutricional en los Hospitales se considera como referencia: La Declaración de Cancún [3] por el Derecho Humano de los enfermos a recibir una terapia nutricional oportuna y óptima en cualquier lugar donde se encuentren que fue emitida por los Presidentes de las Sociedades y Asociaciones de Nutrición Clínica y Terapia Nutricional de América Latina y el Caribe, en el marco del XI Congreso de FELANPE/XV Congreso de AMAEE, en la Ciudad de Cancún Quintana Roo, México, el día 20 de mayo de 2008, la cual menciona entre algunos de sus puntos los siguientes:

a) La atención nutricional no es una opción, es una necesidad impostergable para todo paciente. El soporte nutricional es obligatorio para todo paciente malnutrido o en riesgo. La finalidad del mismo es brindar y satisfacer las necesidades del organismo, aportando los nutrientes precisos cuantitativa y cualitativamente en forma oportuna.

b) Todo paciente hospitalizado debe ser evaluado dentro de las primeras 24 a 48 horas, con un método de tamizaje validado, y de acuerdo a su resultado deberá ser derivado para evaluación nutricional formal.

c) El cuidado nutricional del paciente hospitalizado y la administración del soporte nutricional requieren de la implementación de Guías de Práctica Clínica que contemplen:

i) El método de tamizaje nutricional en todos los hospitales.

ii) Evaluación metabólica-nutricional formal.

iii) Implementación del plan de alimentación y/o terapia nutricional.

iv) Programa de seguimiento.

v) Reevaluación y reformulación del plan y/o terapia o su finalización [3].

\section{Diagnóstico del estado Nutricional}

El diagnóstico del estado nutricional es fundamental en todo paciente y debe seguir una secuencia de evaluación que se inicia con la detección inicial de si hay o no riesgo de malnutrición mediante un cribado nutricional.

La Sociedad Europea de Nutrición Clínica y Metabolismo (ESPEN) define el Riesgo Nutricional como "Las Posibilidades de mejorar o que se agrave el proceso de enfermedad en relación al estado nutricional y metabólico real o potencial [8]. Y al Cribado o screening nutricional como un "proceso rápido y simple realizado por personal de admisión o equipos de atención comunitaria" [9]. Existen diversos trabajos publicados como el de Bozzeti y colaboradores [8] en el cual se realiza screening nutricional a pacientes oncológicos mediante el Método del RNS 2002. 


\section{NRS 2002}

El Nutritional Risk Screening (NRS) 2002 fue desarrollado por Kondrup y un grupo de trabajo de ESPEN en el año 2002 [10], diseñado para incluir medidas tanto de desnutrición potencial como de severidad de la enfermedad. Fue validado contra 128 ensayos controlados para evaluar si era capaz de distinguir a aquellos pacientes con un resultado clínico positivo debido a la intervención nutricional de aquellos que no mostraron beneficio al recibir apoyo nutricional.

Posteriormente, un ensayo prospectivo, controlado con 212 pacientes hospitalizados utilizando el NRS 2002 mostró un aumento de la ingesta nutricional en pacientes que recibieron intervención nutricional cuando fueron catalogados como en riesgo nutricional y una estancia hospitalaria más corta en pacientes con complicaciones como infecciones en relación a los que no recibieron terapia nutricional [11].

El propósito de la evaluación nutricional es predecir la probabilidad de un mejor o peor resultado debido a factores nutricionales, y si es probable que el tratamiento nutricional influya en esto. El resultado del tratamiento se puede evaluar de varias maneras [10]:

1. Mejora o, al menos, previene el deterioro de la función mental y física.

2. Número reducido o disminución de la gravedad de las complicaciones de la enfermedad o su tratamiento.

3. Recuperación acelerada de la enfermedad y acortamiento de la convalecencia.

4. Reducido consumo de recursos, por ejemplo: Duración de la estancia hospitalaria y otras prescripciones.

Por lo tanto, el deterioro nutricional identificado por el examen debe ser relevante para estos objetivos y resultados y puede variar según las circunstancias, edad o tipo de enfermedad, entre otras. En la comunidad, la desnutrición, con o sin enfermedad crónica, puede ser el factor principal que determina la función mental o física de un individuo. En otros ámbitos como, en el hospital o un asilo de ancianos, los factores de la enfermedad asumen una mayor importancia con la desnutrición asociada a la enfermedad, asumiendo un rol importante aunque sea papel secundario.

Por lo tanto, la evaluación en la comunidad se puede enfocar principalmente en variables nutricionales basadas en los resultados de estudios de semi-hambre como los de Ancel Keys y sus colegas en 1950 [12]. En los hospitales, otros aspectos de la enfermedad deben considerarse en combinación con mediciones puramente nutricionales para determinar si es probable que el apoyo nutricional sea beneficioso. Los ensayos controlados aleatorios de apoyo nutricional en grupos de enfermedades particulares pueden por lo tanto, proporcionar importante evidencia en base al riesgo nutricional [13].

El propósito del sistema NRS-2002 es detectar la presencia de desnutrición y el riesgo de desarrollar desnutrición en el entorno hospitalario [10]. Contiene los componentes nutricionales de MUST [14] y además, una clasificación de la gravedad de la enfermedad como un reflejo del aumento de los requisitos nutricionales. Incluye cuatro preguntas como parte del paso previo a otra evaluación. Con los prototipos para la gravedad de la 
enfermedad dados, se pretende cubrir todas las categorías posibles de pacientes en un hospital. Un paciente con un diagnóstico particular no siempre pertenece a la misma categoría. Un paciente con cirrosis, por ejemplo, que ingresa en cuidados intensivos debido a una infección grave, debe recibir una puntuación de 3, en lugar de 1. También incluye la vejez como un factor de riesgo.

Además el NRS 2002 por sus características puede ser realizado tanto por Médicos, como otros miembros del equipo de Salud como Enfermeras, Nutricionistas, Auxiliares de Enfermería. Consta de 2 partes un screening inicial y uno final. (Figura 1 y 2).

Figura 1. Valoración inicial del riesgo nutricional.

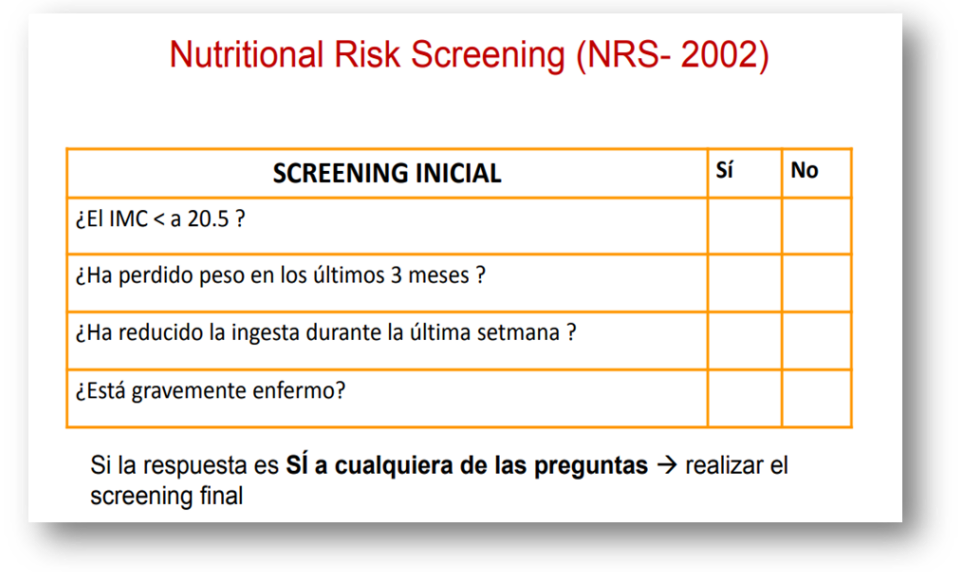

Figura 2. Valoración Final del riesgo nutricional 
1) Escoger la puntuación de Alteración del estado nutricional (la variable con más puntuación) y la Severidad de la enfermedad

2) Sumar las dos puntuaciones. Si el paciente es mayor a 70 años agregar un punto al valor final.

3) Si el puntaje es mayor a 3 iniciar un Plan Nutricional.

4) Si el puntaje es menor a 3 , se recomienda repetir el screening semanalmente, con la finalidad por ejemplo, que si el paciente va a ser intervenido quirúrgicamente con cirugía mayor se debe realizar un plan de cuidado nutricional preventivo, con el fin de evitar cualquier condición de riesgo asociada.

\section{Valoración Global Subjetiva generada por el paciente}

Se trata de un método diseñado por Detsky y colaboradores en 1987, en el Hospital General de Toronto, para estimación del estado nutricional a través de la historia clínica y la exploración física. Este método de evaluación del estado nutricional tiene mayor sensibilidad y especificidad que la valoración a través de medición de albúmina, transferrina, pruebas de sensibilidad cutánea, antropometría, índice de creatinina-altura o el índice nutricional pronóstico. La Valoración Global Subjetiva (VGS) puede ser utilizada para predecir qué pacientes requieren una intervención nutricional y cuáles se beneficiarían de un soporte nutricional intensivo.

Los datos obtenidos de la historia clínica serían: evolución del peso, ingesta dietética actual en relación con la ingesta habitual del paciente, síntomas digestivos presentes en las últimas dos semanas, capacidad funcional y los requerimientos metabólicos (Figura 3).

Dentro del examen físico se evalúa la pérdida de grasa subcutánea, musculatura y la presencia de edema o ascitis. Cada uno de los apartados se valora como leve, moderado o severo y con los datos de todos ellos se clasifica a los pacientes en tres grupos:

- CATEGORÍA A: Pacientes con un adecuado estado nutricional.

- CATEGORÍA B: Sospecha de malnutrición o malnutrición moderada.

- CATEGORÍA C: Pacientes que presentan una malnutrición severa.

Entre todos los datos recogidos, los parámetros más relevantes en el resultado final van a ser la pérdida de peso, la ingesta dietética y la pérdida de músculo o tejido subcutáneo. A los datos obtenidos de la historia clínica se les otorga un valor algo superior al de los derivados de la exploración física (60\% frente a un $40 \%$ ). Este test ha sido validado en pacientes postratamiento quirúrgico o trasplante y en pacientes $\mathrm{VIH}$. Posteriormente se han hecho dos modificaciones a la VGS inicial para que resulte aplicable a pacientes con cáncer [15] y se ha diseñado la Valoración Global Subjetiva generada por el Paciente (VGS-GP)

En la VGS-GP se involucra al propio paciente en la evaluación, de forma que éste cumplimenta la primera parte del cuestionario, que está referida a la historia clínica, mientras que el médico rellena el resto de la valoración, que es la parte que hace referencia 
a los signos físicos. De esta forma se consigue, además, disminuir el tiempo necesario para su realización. Ésta es una diferencia básica con el VGS tradicional, donde es el médico el encargado de completar todo el cuestionario, recogiendo los datos de historia de la información que le da el paciente. Este cuestionario puede realizarse en todos los ámbitos médicos, tanto en pacientes hospitalizados como ambulantes, bien en las consultas externas como en el propio domicilio del paciente. La utilización sistemática de este método permite identificar a los pacientes con malnutrición y valorar los resultados de las intervenciones nutricionales aplicadas en ellos [16].

La pérdida de peso es uno de los parámetros más relevantes de la escala y se obtiene restando el peso actual del peso habitual tres meses antes. El médico debe incluir datos sobre el diagnóstico oncológico principal, enfermedades concomitantes y tratamiento oncológico planeado con el fin de valorar el riesgo nutricional del mismo. También se deben enumerar otros tratamientos asociados, como los corticoides o anabolizantes, valores de albúmina o prealbúmina, según el caso, y datos de la exploración física centrada en la pérdida de tejido adiposo y masa muscular y la presencia de edemas o ascitis [17].

La VGS-SP resulta muy práctica y permite detectar en mayor porcentaje las patologías oncológicas con mayor prevalencia de déficit nutricional [18], tal como lo reportado en un estudio realizado con 66 pacientes oncológicos en el que se identificaron $55 \%$ de pacientes desnutridos a través de la VGS-SP versus 27 \% identificados usando sólo el Índice de Masa Corporal [18]. Debido a que existen varios reportes [19] que muestran el éxito del uso de esta herramienta, ésta se ha modificado para ser aplicada en diversas patologías [20]. 
Figura 3. Escala de Valoración Global Subjetiva Generada por el Paciente (VGS-SP)

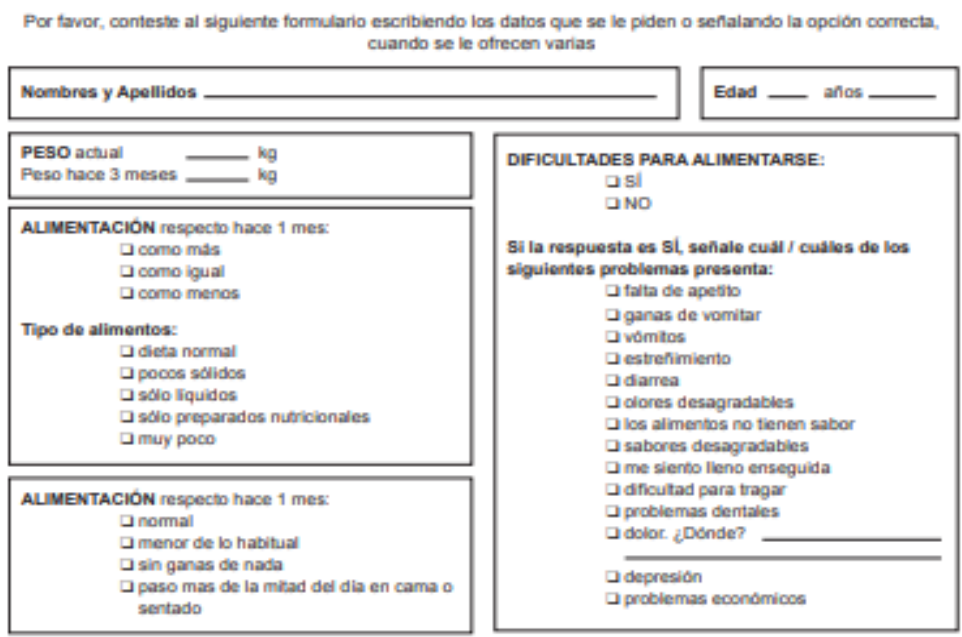

Muchas Gracias. A partir de aqui, lo completara su medico.
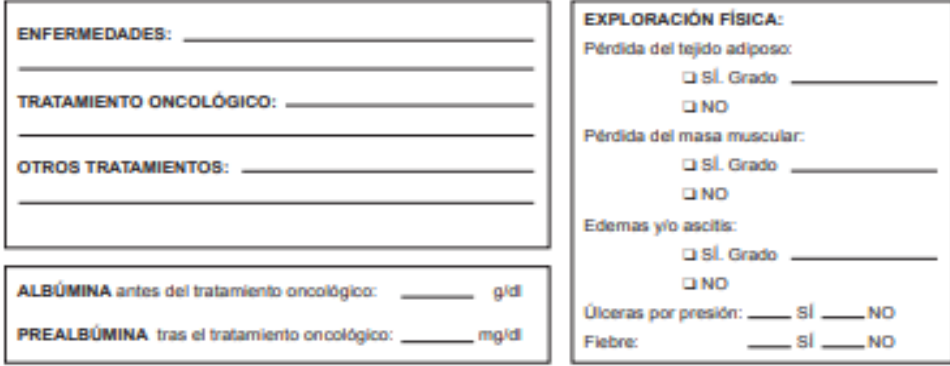

\section{Conclusiones}

Es necesario establecer una política intrahospitalaria para el diagnóstico temprano y oportuno de la desnutrición en los pacientes oncológicos con una prueba de cribado rápida como el test de 4 preguntas NSR-2002. La valoración global subjetiva de los pacientes cribados nutricionalmente debe incluir la cuantificación de la pérdida de peso, la ingesta dietética y la pérdida de músculo o tejido subcutáneo.

\section{Agradecimientos}

Reconocemos a las personas que ayudaron a la compilación bibliográfica de los artículos en la presente revisión, en especial al personal de la biblioteca del Instituto Oncológico Nacional "Dr. Juan Tanca Marengo", Solca-Guayaquil- Ecuador. 


\section{Información adicional}

Nota del Editor

La Revista Oncología Ecu

permanece neutral con respecto a los reclamos jurisdiccionales en mapas publicados y afiliaciones institucionales.
Abreviaturas

NSR-2002: Nutritional Risk Screening 2002.

VGS-SP: Valoración Global Subjetiva Generada por el Paciente.

ESPEN: Sociedad Europea de Nutrición Clínica y Metabolismo.

\section{Archivos Adicionales}

Ninguno declarado por los autores.

\section{Fondos}

Los fondos de la investigación fueron propios de los autores del presente artículo.

\section{Disponibilidad de datos y materiales}

Existe la disponibilidad de los artículos consultados en la presente revisión.

\section{Contribuciones de los autores}

Todos los autores participaron por igual en la concepción de la idea de revisión, recolección de artículos, escritura del artículo. Todos los autores leyeron y aprobaron la versión final del artículo.

\section{Información de los autores}

Dolores Rodríguez Veintimilla, Doctara Jefe del Servicio de Nutrición Clínica y Dietética del Instituto Oncológico Nacional. SOLCA- matriz Guayaquil E-mail: dra.rodriguezv@yahoo.com (iD) https://orcid.org/0000-0001-7030-0461

Evelyn Frías-Toral, Máster en Investigación Clínica y Epidemiológica, Diplomado Nutrición Enteral y Parenteral. Coordinadora del Comité de Investigación del Instituto Oncológico Nacional. SOLCA- matriz Guayaquil E-mail: evelynft@gmail.com

Sergio Santana Portobén, Médico del Hospital Pediátrico Docente Juan Manuel Marquez de La Habana. Editor-Ejecutivo de la Revista Cubana de Alimentación y Nutrición. Cuba. Email: ssergito@infomed.sld.cu. 
Aprobación de ética y consentimiento para participar

No aplica a un artículo de revisión.

\section{Consentimiento para publicación}

No aplica.

\section{Referencias}

1. Pressoir M, Desne S, Berchery D, Rossignol G, Poiree B, Meslier M, et al. Prevalence, risk factors and clinical implications of malnutrition in French Comprehensive Cancer Centres. $\mathrm{Br} \mathrm{J}$ Cancer 2010;102(6):966e71.

2. WieGA,ChoYA,KimSY,KimSM,BaeJM,JoungH.Prevalence and risk factors of malnutrition among cancer patients according to tumor location and stage in the National Cancer Center in Korea. Nutrition 2010;26(3):263e8.

3. Castillo Pineda JC, Figueredo Grijalva R, Dugloszewski C, Díaz Reynoso RJA, Spolidoro Noroña JV, Matos A, et al. Declaración de Cancún: Declaración internacional de Cancún sobre el derecho a la nutrición en los hospitales. Nutr Hosp (Madrid). 2008;19(1):12-19.

4. GBD 2015 Risk Factors Collaborators. Global, regional, and national comparative risk assessment of 79 behavioural, environmental and occupational, and metabolic risks or clusters of risks, 1990-2015: a systematic analysis for the Global Burden of Disease Study 2015. Lancet. 2016 Oct; 388 (10053):16591724

5. Plummer M, de Martel C, Vignat J, Ferlay J, Bray F, Franceschi S. Global burden of cancers attributable to infections in 2012: a synthetic analysis. Lancet Glob Health. 2016;4(9):e609-16. DOI: 10.1016/S2214$109 \times(16) 30143-7$.

6. Boffetta, P., Couto, E., Wichmann, J., Ferrari, P., Trichopoulos, D., Bueno-de-Mesquita, H.B. et al. Fruit and vegetable intake and overall cancer risk in the European prospective investigation into cancer and nutrition (EPIC). J Natl Cancer Inst. 2010;102:529-537

7. Forman, D. Sierra, M. Cancer in Central and South America: Introduction. Cancer Epidemiology 2016;S3S10.

8. Instituto Nacional del Cáncer. Diccionario del cáncer. https://www.cancer.gov/espanol/publicaciones/diccionario/def/terapia-nutricional-medica.

9. Lochs H, Allison S, Meier R, Pirlich M, Kondrup J, Schneider S, et al. Introductory to the ESPEN Guidelines on Enteral Nutrition: Terminology, Definitions and General Topics. Clin Nutr. 2006;25(2):180-186.

10. Kondrup J, Rasmussen HH, Hamberg O, Stanga Z, Ad Hoc ESPEN Working Group (2003) Nutritional risk screening (NRS): a new method based on an analysis of controlled clinical trials. Clin Nutr. 2002;22: $321-336$.

11. Johansen $\mathrm{N}$, Kondrup J, Plum L. Effect of nutritional support on clinical outcome in patients at nutritional risk. Clin Nutr. 204;23:539-550.

12. Keys A, Brozek J, Henschel A et al. The Biology of Human Starvation. Minneapolis: University of Minnesota Press; 1950; p. 703-748, 819, 918.

13. Rabito E, Marcadenti A, da Silva Fink J, Figueira L, Silva F. Nutritional Risk Screening 2002, Short Nutritional Assessment Questionnaire, Malnutrition Screening Tool, and Malnutrition Universal 
Screening Tool Are Good Predictors of Nutrition Risk in an Emergency Service. Nutrition in Clinical Practice. 20017;32:526-532. DOI:10.1177/0884533617692527

14. Elia M. Screening for malnutrition: A multidisciplinary responsibility. Development and Use of the Malnutrition Universal Screening Tool ('MUST') for Adults. Redditch: BAPEN; 2003.

15. Detsky AS, Mc Laughlin JR, Baker JP, Johnson N, Whittaker S, Mendelson RA, Jeegeebhoy KN. What is subjective global assessment of nutritional status? J Parenter Enteral Nutr. 1987;11:8-13

16. Ottery DF. Rethinking nutritional support of the cancer patient: the new field of nutritional oncology: Seminars in Oncology. 1994;21:770-8.

17. Cerezo, L. Diagnóstico del estado nutricional y su impacto en el tratamiento del cáncer. Oncolog. 2005; 28(3):23-28.

18. Persson C. The Swedish version of the patients generated subjective global assessment of nutritional status: gastrointestinal vs. urological cancers. Clin Nutr. 1999;18:71-7.

19. Gómez C. Valoración Global Subjetiva en el paciente neoplásico. Nutr Hosp. 2003;18 353-357.

20. Wu B. Validation of the Chinese version of the Subjective Global Assessment Scale of nutritional status in a sample of patients with gastrointestinal cancer. Int J Nurs Stud. 2010;47:323-331. 